\title{
Posibles impactos de la carretera lquitos-Saramiriza sobre la biodiversidad de Loreto, Perú
}

\section{Possible impacts of the lquitos-Saramiriza highway on the biodiversity of Loreto, Peru}

Leonardo Maffei*.

https://orcid.org/0000-0001-5318-4956

leomaffei@yahoo.com

\section{E. Daniel Cossios}

https://orcid.org/0000-0003-4188-7632 dcossios@yahoo.com

\section{*Corresponding author}

Biosfera Consultores Ambientales, Calle Las Fresas 730 Miraflores, Lima, Perú.

\section{Citación}

Maffei L, Cossios ED. 2021. Carretera Iquitos-Saramiriza: posibles impactos sobre la biodiversidad de Loreto, Perú. Revista peruana de biología 28(especial): e21963 303- 314 (Diciembre 2021). doi: http://dx.doi. org/10.15381/rpb.v28iespecial.21963

\section{Presentado: $\quad 09 / 02 / 2021$

Aceptado: $\quad 10 / 08 / 2021$ \\ Publicado online: $30 / 12 / 2021$}

Editor:

Leonardo Romero

\section{Resumen}

La construcción de la carretera lquitos-Saramiriza proyectada en el departamento de Loreto plantea amenazas sobre la fauna y flora de una zona altamente biodiversa y poco alterada. Aquí, resumimos dichas amenazas en tres puntos: 1) impactos probables, identificados a través de revisión de literatura, 2) estimación de la deforestación, realizada por comparación con otras carreteras amazónicas peruanas y 3) especies más probablemente impactadas. La lista de impactos probables incluyó fragmentación de hábitat, deterioro ambiental, atropellos, dispersión de patógenos, especies invasoras, deforestación, caminos no oficiales y cacería. Estos impactos podrían afectar a unas 3961 especies de plantas y 1595 de vertebrados. La deforestación, en el transcurso de las tres próximas décadas, podría llegar a ser entre 1128 y $3200 \mathrm{~km}^{2}$, lo que equivale a más del $80 \%$ de la deforestación que ha sufrido Loreto en los últimos 20 años. La fragmentación podría afectar principalmente a unas 83 especies arborícolas y ampliarse hasta la totalidad de fauna y flora en áreas con mayor deforestación. Los atropellos impactarían principalmente a unas 287 especies de animales terrestres, mientras la cacería podría dirigirse hacia unas 31 especies principales. Para 14 especies de vertebrados, la carretera corta completamente su distribución conocida, pudiendo generar poblaciones con poco o nulo intercambio genético. En base a los posibles impactos proyectados, proponemos acciones de mitigación, incluyendo el remplazo de un tramo por transporte fluvial, creación de áreas de protección estricta, instalación de pasos de fauna y la consideración de impactos indirectos en el EIA correspondiente y en otros instrumentos ambientales.

\section{Abstract}

La construcción de la carretera lquitos-Saramiriza proyectada en el departamento de Loreto plantea amenazas sobre la fauna y flora de una zona altamente biodiversa y poco alterada. Aquí, resumimos dichas amenazas en tres puntos: 1) impactos probables, identificados a través de revisión de literatura, 2) estimación de la deforestación, realizada por comparación con otras carreteras amazónicas peruanas y 3) especies más probablemente impactadas. La lista de impactos probables incluyó fragmentación de hábitat, deterioro ambiental, atropellos, dispersión de patógenos, especies invasoras, deforestación, caminos no oficiales y cacería. Estos impactos podrían afectar a unas 3961 especies de plantas y 1595 de vertebrados. La deforestación, en el transcurso de las tres próximas décadas, podría llegar a ser entre 1128 y $3200 \mathrm{~km}^{2}$, lo que equivale a más del $80 \%$ de la deforestación que ha sufrido Loreto en los últimos 20 años. La fragmentación podría afectar principalmente a unas 83 especies arborícolas y ampliarse hasta la totalidad de fauna y flora en áreas con mayor deforestación. Los atropellos impactarían principalmente a unas 287 especies de animales terrestres, mientras la cacería podría dirigirse hacia unas 31 especies principales. Para 14 especies de vertebrados, la carretera corta completamente su distribución conocida, pudiendo generar poblaciones con poco o nulo intercambio genético. En base a los posibles impactos proyectados, proponemos acciones de mitigación, incluyendo el remplazo de un tramo por transporte fluvial, creación de áreas de protección estricta, instalación de pasos de fauna y la consideración de impactos indirectos en el EIA correspondiente y en otros instrumentos ambientales.

Palabras clave:

Cacería, deforestación, desarrollo, fauna, flora, fragmentación.

Keywords:

Deforestation, development, flora, fragmentation, hunting, wildlife . 


\section{Introducción}

La Amazonía es una de las regiones con mayor diversidad biológica del planeta y con mayor número de endemismos (Finer et al. 2008), además de poseer la superficie de bosque continuo más grande del mundo (Ceballos \& Enrich 2006; Kileen et al. 2007). Esta biodiversidad soporta varias amenazas, muchas de ellas asociadas a proyectos de gran o pequeña escala, que incluyen la pérdida de bosque debido al avance de la frontera agrícola y ganadera, la tala indiscriminada para uso de madera, la apertura de nuevas carreteras, la cacería comercial y la construcción de represas hidroeléctricas, entre las más importantes (Soares et al. 2006, Finer et al. 2008).

Se ha estimado que, de continuar su ritmo actual, la práctica de tala y quema de bosques con el objeto de incrementar la superficie disponible para cultivos agrícolas y pastoreo de ganado llevaría a que los bosques amazónicos se vean reducidos al 53\% de su extensión original para el año 2050 (Soares et al. 2006). Además del evidente impacto que tiene sobre el paisaje, el cambio en la cobertura vegetal a largo plazo, causado por la deforestación, es la causa de origen humano que más contribuye con la pérdida de riqueza de especies en la Amazonía (Howard et al. 2020). Otras amenazas importantes a la biodiversidad amazónica son la cacería en todas sus formas, el tráfico de especies silvestres y la invasión de especies exóticas, junto con las enfermedades que estas pueden introducir en las especies nativas (SERFOR 2018, Howard et al. 2020).

Entre los factores desencadenantes de deforestación amazónica destacan las carreteras. Este tipo de infraestructura mejora la comunicación entre pueblos y facilita el transporte de materias primas y productos, pero puede también generar amenazas a la biodiversidad, que dependen del contexto social de los países en los que son construidas (Fearnside 2015). En la cuenca amazónica, la presencia de carreteras es uno de los factores que mejor predice la deforestación, como en Brasil (Pfaff et al. 2007, Soares et al. 2006), Perú (Imbernon 1999), Bolivia (Kaimowitz 1997) y Ecuador (Mena et al. 2006). Estas vías de comunicación facilitan la deforestación y la cacería al promover la colonización (Perz 2014, Gallice et al. 2017), haciendo parte de un círculo vicioso en el que la mayor presencia de migrantes justifica la aparición de nuevas carreteras y caminos, que incrementan el valor monetario de los terrenos y atraen nuevos colonizadores (Fearnside 1987). Por otro lado, las carreteras tienen un efecto fragmentador del hábitat y pueden tener un impacto negativo sobre las redes de arroyos y la calidad del agua, así como facilitar la cacería, fomentar la propagación de especies exóticas invasoras y acelerar el cambio climático local (Trombulak y Frissell 2000), además de tener posibles consecuencias sociales sobre las poblaciones locales, como conflictos entre las comunidades locales o indígenas y los posibles invasores, tráfico de tierras o la inserción de actividades delictivas (Perz 2014).

El 04 de octubre del año 2017, el Estado peruano promulgó la Ley $\mathrm{N}^{\circ} 30670$ que declara de necesidad pública e interés nacional la construcción de la carretera Iquitos-
Santa María de Nanay-Andoas-Saramiriza (carretera Iquitos-Saramiriza). Esta carretera proyecta tener una extensión de $688 \mathrm{~km}$ y atravesar territorios de selva baja actualmente en buen estado de conservación, situados en el noroeste de la región peruana de Loreto.

El presente artículo estima los impactos de la carretera proyectada Iquitos-Saramiriza sobre la biodiversidad de Loreto considerando los siguientes puntos: a) presentación de los principales probables impactos de la carretera, b) estimación de la deforestación que podría generarse, a partir de la comparación con otras carreteras amazónicas peruanas y c) listado de las especies de fauna y flora con mayor probabilidad de ser impactadas por el proyecto. Finalmente, presentamos recomendaciones, en lo que respecta a biodiversidad, para ser tomadas en futuros estudios de impacto ambiental y del monitoreo de la biodiversidad, de tal manera que el presente análisis brinde un insumo que guie acciones para la mitigación de los impactos en el caso se llegue a concretar el proyecto.

\section{Material y métodos}

Área de desarrollo de la carretera Iquitos-Saramiriza. - El recorrido proyectado de la carretera IquitosSaramiriza se extiende en la región noroeste del departamento de Loreto, en la ecorregión de selva baja (Fig. 1). La carretera inicia aproximadamente en el kilómetro 59 de la carretera Iquitos-Nauta (UTM WGS84: 669310/9538960). Primero se dirige hacia el norte recorriendo $72 \mathrm{~km}$ de la provincia de Maynas, luego $342 \mathrm{~km}$ de la provincia de Loreto y, finalmente, $274 \mathrm{~km}$ de la provincia de Alto Amazonas; luego se dirige al sur-oeste, hasta Saramiriza (UTM WGS84: 231658/9494727), totalizando $688 \mathrm{~km}$. En ese recorrido, atraviesa 19 ríos (Tabla 1), y las poblaciones de Nuevo Andoas, Puerto Alegría, Borja y Saramiriza.

Tabla 1. Ríos cruzados por el trazo proyectado de la carretera Loreto-Saramiriza.

\begin{tabular}{lcc}
\hline Provincia & Río cruzado & $\begin{array}{c}\text { Distancia al inicio de la } \\
\text { carretera }(\mathrm{en} \mathrm{km})\end{array}$ \\
\hline \multirow{2}{*}{ Loreto } & Tigre & 154 \\
& Corrientes & 387 \\
& Macurasi & 409 \\
\hline \multirow{3}{*}{ Alto Amazonas } & Pastaza & 438 \\
& Huasaga & 479 \\
& Huitoyacu & 521 \\
& Morona & 631 \\
& Marañón & 666 \\
\hline
\end{tabular}

Identificación de posibles impactos. - Para identificar los posibles impactos ambientales de la carretera Iquitos-Saramiriza sobre la biodiversidad, se revisó la literatura relacionada con el tema partiendo de la base que es de esperar que los impactos comunes a las carreteras en general y a las amazónicas en particular se den también en la carretera Iquitos-Saramiriza y en una magnitud similar a la de otras carreteras amazónicas 
peruanas. La búsqueda de información se realizó en internet con el buscador Google Scholar y usando las palabras clave "carretera" o "Amazonía" en combinación con las palabras "impacto", "EIA", “colonización”, "cacería” y "zoonosis", tanto en castellano como en inglés. Además, se buscó publicaciones que fueron citadas como importantes en la literatura que ya se había encontrado.

Estimación de la deforestación. - Dado que los impactos de una carretera sobre el bosque dependen de las condiciones políticas y sociales del país en el que es construida (Fearnside 2015), se estimó la deforestación probable asociada a la carretera Iquitos-Saramiriza a partir de la deforestación ocurrida alrededor de otras dos carreteras existentes en la Amazonía peruana: la carretera Iquitos-Nauta, situada también en el departamento de Loreto, y la carretera Interoceánica, en el departamento de Madre de Dios. Para la carretera Iquitos-Nauta, se evaluó $75 \mathrm{~km}$ de esta vía, desde Nauta hasta el centro poblado Varillal, $21 \mathrm{~km}$ al sur de Iquitos. En el caso de la carretera Interoceánica se evaluó un total de 310 km, compuestos por $223 \mathrm{~km}$ del sector Iñapari-Puerto Maldonado, y $87 \mathrm{~km}$ desde Puerto Maldonado hacia el oeste, en la frontera con Brasil, dejándose de lado el ambiente urbano (la ciudad de Puerto Maldonado) y el sector conocido como "arco minero", en el que se ha desarrollado una particular actividad de extracción de oro que no es aplicable al caso de esta publicación. A lo largo de esas dos carreteras, se midieron los caminos secundarios o no oficiales creados a partir de la vía principal usando imágenes satelitales de libre disponibilidad en Google Earth®. Además, se estimó la pérdida total de bosque hasta $20 \mathrm{~km}$ de distancia a cada lado, así como la pérdida anual de bosque durante el periodo 2001-2018 con información de cobertura y pérdida de bosque disponibles en http://geobosques.minam.gob.pe/. Se analizaron imágenes Landsat 7 para los años 2001-2011 y con imágenes Landsat 5 para el periodo 2012-2018, en ambos casos con una resolución espacial de 30 metros y con 8 bandas espectrales. El procesamiento de datos fue desarrollado con el programa ArcGIS versión 10.3.

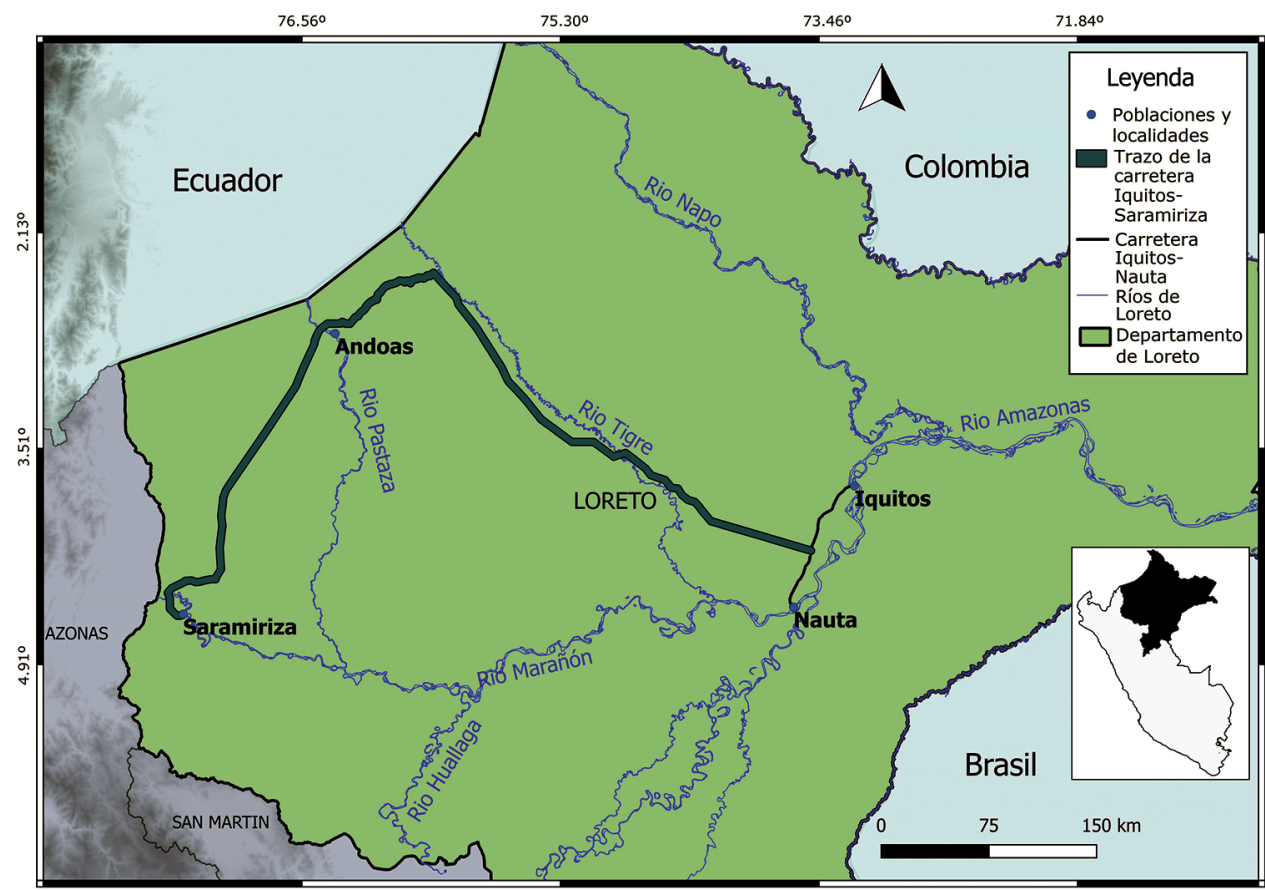

Figura 1. Posición del trazo de la carretera proyectada lquitos-Saramiriza, en el departamento peruano de Loreto.

Listado de especies más susceptibles a impactos. - Para definir qué especies de vertebrados y de plantas vasculares tienen mayor probabilidad de ser afectadas por la carretera Iquitos-Saramiriza, se contabilizó las especies que han sido registradas a lo largo del trazo planeado hasta una distancia de $5 \mathrm{~km}$ y hasta $20 \mathrm{~km}$ a cada lado. Con el fin de ampliar el número de especies registradas, debido a que las evaluaciones de fauna y flora realizadas en esa zona han tenido un esfuerzo limitado, se confeccionó además una lista de las especies registradas en Loreto en la toda la región ubicada al oeste del río Napo y al norte del río Marañón - región donde se abriría esta carretera. Los datos georreferenciados de los registros de especies provinieron de otros trabajos presentados en este volumen por Zárate-Gómez et al. (en revisión) para las plantas, Meza-Vargas et al. (en revisión) para los peces, Aguilar et al. (en revisión) para los anfibios, Aguilar (en revisión) para los reptiles, Salinas (en revisión) para las aves y Sánchez y Pacheco (en revisión), Graham y Pacheco (en revisión) y Díaz y Pacheco (en revisión) para los mamíferos. Dada la ausencia de barreras aparentemente importantes entre los ríos principales, todas las especies registradas en ese espacio fueron tomadas en cuenta como de presencia probable en el área de influencia de la carretera y, basándonos en sus hábitos, se realizó una lista de especies que podrían ser afectadas por cada posible impacto identificado. 
Con el fin de conocer si la cantidad de especies registradas hasta $20 \mathrm{~km}$ de distancia del trazo de la vía había alcanzado la asíntota de acumulación (Pielou 1969), se elaboraron curvas de acumulación de especies basadas en la cantidad de registros obtenidos por año en cada interfluvio. Con estos datos de abundancia se calcularon los indicadores de ACE y Chao 1 con el programa EstimateS 9.1.0 (Colwell 2013) para obtener una estimación de la riqueza probable.

\section{Resultados}

Impactos. - La revisión de literatura permitió identificar los siguientes impactos probables:

- Fragmentación del hábitat. La fragmentación sucede cuando el hábitat se divide en dos o más partes entre las cuales el tránsito de individuos se reduce o se interrumpe. La simple presencia de una carretera fragmenta el hábitat, pero esta fragmentación puede agravarse al avanzar la deforestación a lo largo de la vía. El aislamiento puede afectar a especies de costumbres arborícolas como primates y algunas especies de reptiles, que sufren la interrupción del dosel y a especies que se dispersan a nivel del suelo, como anfibios o pequeños mamíferos (Valladares-Padua et al. 1995, Jochimsem et al. 2004, Goosem 2002). Los efectos de la fragmentación se agravan a medida que avanza la deforestación, como con la apertura de caminos secundarios, y mientras más ancha sea la banda deforestada. Si la deforestación a lo largo de la carretera se diera en una banda de ancho suficiente, esta podría limitar también el paso de mamíferos voladores y aves (Fensome \& Mathews 2016, Khamcha et al. 2018) y afectar la dispersión y la polinización de plantas (Marjakangas et al. 2019, Volpe et al. 2016).

- Cambios físicos del ambiente terrestre circundante. Se identificaron cinco características físicas ambientales que son modificadas por las carreteras y que pueden aumentar o disminuir el paso de organismos por el espacio que estas ocupan: densidad y humedad del suelo, temperatura, ingreso de luz, polvo y acumulación de agua (Trombulak y Fissel 2000). Los cambios en las características físicas en la zona de la carretera pueden actuar de diferente forma. El calor acumulado en el suelo, por ejemplo, es liberado de noche y algunas especies, principalmente aves y reptiles, responden a estas zonas de calor acercándose, lo que aumenta la probabilidad de que sean atropelladas (e.g. Whitford 1985, Heigl et al. 2017). En el caso del polvo que se levanta durante la construcción, las partículas que se depositan sobre la vegetación, hasta $1 \mathrm{~km}$ de distancia y dependiendo de su tamaño, pueden reducir la capacidad de crecimiento de las plantas (Farmer 1993).

- Cambios en ambientes acuáticos. Durante la construcción, la extracción de grava y arena puede tener un efecto importante sobre la fauna acuática al destruir sitios de puesta de huevos, lugares de alimentación e incrementando la turbidez; el mantenimiento y limpieza de maquinaria puede llevar contaminación a cuerpos de agua. Durante el funcionamiento, la erosión de carreteras no pavimentadas puede alterar la calidad del agua de riachuelos, pantanos y lagunas aledañas (Ledec \& Posas 2003).

- Atropellos.Se ha hecho pocos estudios sobre atropellamiento de fauna silvestre en la Amazonía. Aunque trabajos iniciales se centraron en el impacto de este fenómeno sobre mamíferos mayores (e.g. Cáceres et al. 2010), investigaciones recientes muestran que los atropellamientos en bosques amazónicos afectan a una gran variedad de especies, entre anfibios, reptiles, aves y mamíferos (Brum et al. 2018, Filius et al. 2020).

- Dispersión de patógenos y especies invasoras. Las carreteras sirven para transportar animales domésticos en vehículos de carga $\mathrm{y}$, en las paradas que realizan para vender los mismos, se diseminan también las enfermedades zoonóticas (Gad et al. 1986); para el caso de las plantas, las hierbas invasoras encuentran una vía ideal para dispersar sus semillas cuando son transportadas por los túneles de viento que se forman en las carreteras lineales (Rieux et al. 2014). La dispersión de especies invasoras se ve facilitada por las carreteras y la aparición de ambientes fragmentados, como el caso del coyote (Canis latrans), ejemplo de especie invasora de gran tamaño, ha colonizado América central hasta Panamá, partiendo desde Norteamérica, y está a punto de ingresar en Colombia (Monroy-Vilchiz et al. 2020) usando ambientes antropogénicos para su dispersión como cultivos o zonas con población humana, ambos productos indirectos de las carreteras. Para el caso de las plantas, se reportó en el Valle Kashmir, India, que el $69 \%$ de las plantas invasoras registradas en la zona estaban en las cercanías del sistema carretero, mientras el incremento de especies nativas era proporcional a la distancia a las carreteras (Dar et al. 2015).

- Deforestación. Además de la pérdida de bosque que se da sobre la superficie ocupada por una carretera y en las franjas aledañas, las carreteras fomentan la deforestación al ofrecer acceso a nuevas tierras. Existen varios ejemplos de deforestación asociada a carreteras, como el de Rondonia, Brasil, donde hasta 1990, entre el 16 y el 19\% del territorio del estado habría sido deforestado como consecuencia directa de la construcción de la carretera transamazónica (Frohn et al. 1990), o el de Madre de Dios, Perú, donde la Amazonía está sujeta al impacto de la pavimentación de la carretera Interoceánica, que además de abrir acceso a nuevas áreas para actividades de tala, ha atraído migrantes y generado la pérdida, entre 1999 y 2005, de más de 1000 $\mathrm{km}^{2}$ de selva a lo largo de la carretera (Finer \& Mamani 2020, Oliveira et al 2007).

- Construcción de caminos no oficiales. Los colonizadores suelen abrir nuevos caminos a partir de las carreteras para acceder a nuevas tierras con el fin 
de explotar recursos, como ampliar los cultivos o practicar la tala y a lo largo de carreteras amazónicas, los caminos no oficiales - llamados "caminos pescado", constituyen un efecto secundario de gran impacto (Perz 2007). Como ejemplo, en la Amazonía brasileña la longitud total de los caminos pescado es casi el triple que la de las carreteras oficiales (Laurance et al. 2014). Para el caso de la carretera Iquitos-Nauta, medimos los caminos no oficiales que han sido abiertos a partir de la carretera, e identificamos $164 \mathrm{~km}$ de estos caminos, lo que significa un aumento del $170 \%$ en las vías de ingreso hacia la selva.

- Cacería. Al facilitar el ingreso a zonas antes inaccesibles, las carreteras y los caminos construidos a su alrededor permiten el aumento de la caza, incluso en zonas cercanas a áreas naturales protegidas (Suárez et al. 2009, Espinosa et al. 2018).

Estimación de la deforestación. - La deforestación total a lo largo del tramo evaluado de $75 \mathrm{~km}$ de la carretera Iquitos-Nauta fue estimada en $122.8 \mathrm{~km}^{2}$, lo que equivale a $1.64 \mathrm{~km}^{2}$ por cada kilómetro de carretera. La deforestación anual en el periodo 2001-2018 a lo largo de ese tramo, en una banda de $10 \mathrm{~km}$ de ancho total, fue de $0.55 \%$ en promedio, es decir 609 ha anuales (de $=264$; rango:198-1068 para un área de estudio de $1104 \mathrm{~km}^{2}$ ).

Para la carretera Interoceánica, la deforestación total a lo largo de los $310 \mathrm{~km}$ estudiados fue de $1442 \mathrm{~km}^{2}$, lo que equivale a $4.65 \mathrm{~km}^{2}$ de área deforestada por cada kilómetro de carretera. En el periodo 2001-2018, la deforestación anual fue de $0.84 \%$ hasta $5 \mathrm{~km}$ de distancia de la vía, lo que equivale a 1924 ha anuales en promedio (de=792; rango: 960-3234 para un área de estudio de $2278 \mathrm{~km}^{2}$ ).

Considerando las tasas de deforestación observadas para las carreteras Iquitos-Nauta e Interoceánica, la carretera Iquitos-Saramiriza podría generar una pérdida anual de bosque de entre 37.8 y $57.8 \mathrm{~km}^{2}$ dentro de una franja de hasta $5 \mathrm{~km}$ a cada lado del trazo, lo que significa, en un lapso de 20 años, una pérdida de entre 756 y $1156 \mathrm{~km}^{2}$ o entre el 11 y el $17 \%$ del área de esa franja. Por otro lado, la pérdida total de bosque a lo largo de la carretera podría oscilar entre 1128 y $3200 \mathrm{~km}^{2}$ en un periodo de 20 a 30 años.
Especies en la zona de influencia de la carretera y susceptibles a impactos específicos. - Se consiguieron un total de 3961 registros de especies de plantas y 1595 de vertebrados en el área ubicada entre los ríos Napo y Marañón, considerándose todas ellas especies de presencia probable en el área de influencia de la carretera. De las especies conocidas en Loreto, las registradas en esta zona corresponden al $42 \%$ de las plantas, $75 \%$ de los peces, $76 \%$ de los anfibios, $81 \%$ de los reptiles, $39 \%$ de las aves y $51 \%$ de los mamíferos. De estas especies, 24 plantas y 32 vertebrados son especies amenazadas según las normas peruanas, mientras que 204 plantas y 61 vertebrados son endémicos peruanos. Dentro del área situada hasta los $5 \mathrm{~km}$ de distancia de la carretera -una franja de $10 \mathrm{~km}$ en total-, se registró 1527 especies de plantas y 298 de vertebrados, mientras que hasta los 20 $\mathrm{km}$ de distancia -una franja de $40 \mathrm{~km}$ en total- se registró 2966 especies de plantas y 1150 de vertebrados (Tabla 2 ). Las curvas de acumulación de especies y proyecciones de riqueza sugieren que el número real de especies de mamíferos dentro de los $20 \mathrm{~km}$ de distancia a la carretera proyectada es cercano a lo observado (entre un 4.2 y $6.9 \%$ mayor), mientras que los anfibios, plantas y peces muestran curvas con pendientes elevadas, alejadas de una asíntota, sugiriendo que su riqueza real en el área es mucho más elevada (Fig. 2). En promedio, los índices de ACE y Chao1 mostraron un incremento del $30 \%$ con respecto a la riqueza observada.

Todas las especies registradas en el área de influencia de la carretera son consideradas susceptibles a ser afectadas por la pérdida de hábitat causada por la deforestación. Los organismos más claramente afectados por la fragmentación serían los vertebrados de costumbres principalmente arborícolas (42 especies de mamíferos, 29 anfibios y 12 reptiles registrados en la zona) pero este problema podría ampliarse hasta la totalidad de especies presentes en el área si la magnitud de la deforestación fuera lo suficientemente grande. Los atropellos tendrían un mayor impacto sobre vertebrados de movilización básicamente terrestre (287 especies), mientras la cacería tanto comercial como de subsistencia podría afectar a unas 31 especies de vertebrados (Anexo 1).

Tabla 2. Número de especies de plantas y vertebrados registradas en el área de influencia de la carretera Iquitos-Saramiriza.

\begin{tabular}{|c|c|c|c|c|c|c|c|}
\hline & & Plantas & Peces & Anfibios & Reptiles & Aves & Mamíferos \\
\hline \multirow{3}{*}{$\begin{array}{l}\text { Especies registradas hasta } 5 \\
\mathrm{~km} \text { del trazo de la carretera }\end{array}$} & Especies & 1527 & 161 & 47 & 5 & 5 & 80 \\
\hline & Amenazadas & 6 & - & - & - & - & - \\
\hline & Endémicas & 49 & 10 & 1 & - & - & - \\
\hline \multirow{3}{*}{$\begin{array}{l}\text { Especies registradas hasta } 20 \\
\mathrm{~km} \text { del trazo de la carretera }\end{array}$} & Especies & 2966 & 488 & 118 & 73 & 308 & 163 \\
\hline & Amenazadas & 11 & 2 & 1 & 2 & 4 & 3 \\
\hline & Endémicas & 84 & 24 & - & - & 2 & 1 \\
\hline \multirow{3}{*}{$\begin{array}{l}\text { Especies registradas en } \\
\text { Loreto, entre los ríos Napo y } \\
\text { Marañón }\end{array}$} & Especies & 3961 & 732 & 148 & 149 & 351 & 215 \\
\hline & Amenazadas & 24 & 2 & 4 & 6 & 4 & 18 \\
\hline & Endémicas & 204 & 48 & 4 & 1 & 2 & 6 \\
\hline
\end{tabular}


En el caso de 14 especies de vertebrados (cuatro especies de mamíferos, ocho anfibios y dos reptiles), el trazo de la carretera proyectada corta completamente su distribución conocida, por lo que podría generar dos poblaciones con poco o nulo intercambio genético. En otros 11 casos, la distribución es cortada casi en su totalidad. En la Figura 3 se muestra, a manera de ejemplos, los casos del mono araña grisáceo Ateles belzebuth y el huapo ecuatorial Pithecia aequatorialis, dos primates con un corte total de su distribución.

\section{Discusión}

En la Amazonía, las carreteras y las actividades extractivas asociadas a ellas son la principal causa de deforestación y la amenaza más importante hacia la biodi- versidad. Por esto, debería evitarse su construcción en áreas remotas y con alta diversidad biológica (Laurance et al. 2009). Este es justamente el caso de la carretera Iquitos-Saramiriza, puesto que se extendería en una zona altamente biodiversa, rica en especies amenazadas y especies endémicas peruanas. La investigación en esta área ha sido además escasa, por lo que se espera que el verdadero número de especies en ella sea mayor a lo conocido. Adicionalmente, según Vilela et al. (2020) esta carretera tendría un costo por kilómetro 50\% superior al promedio de las carreteras amazónicas y una ganancia económica neta negativa, siendo el proyecto vial amazónico con mayor pérdida económica de un grupo de 75 proyectos evaluados, por lo que las razones para su construcción serían básicamente políticas.
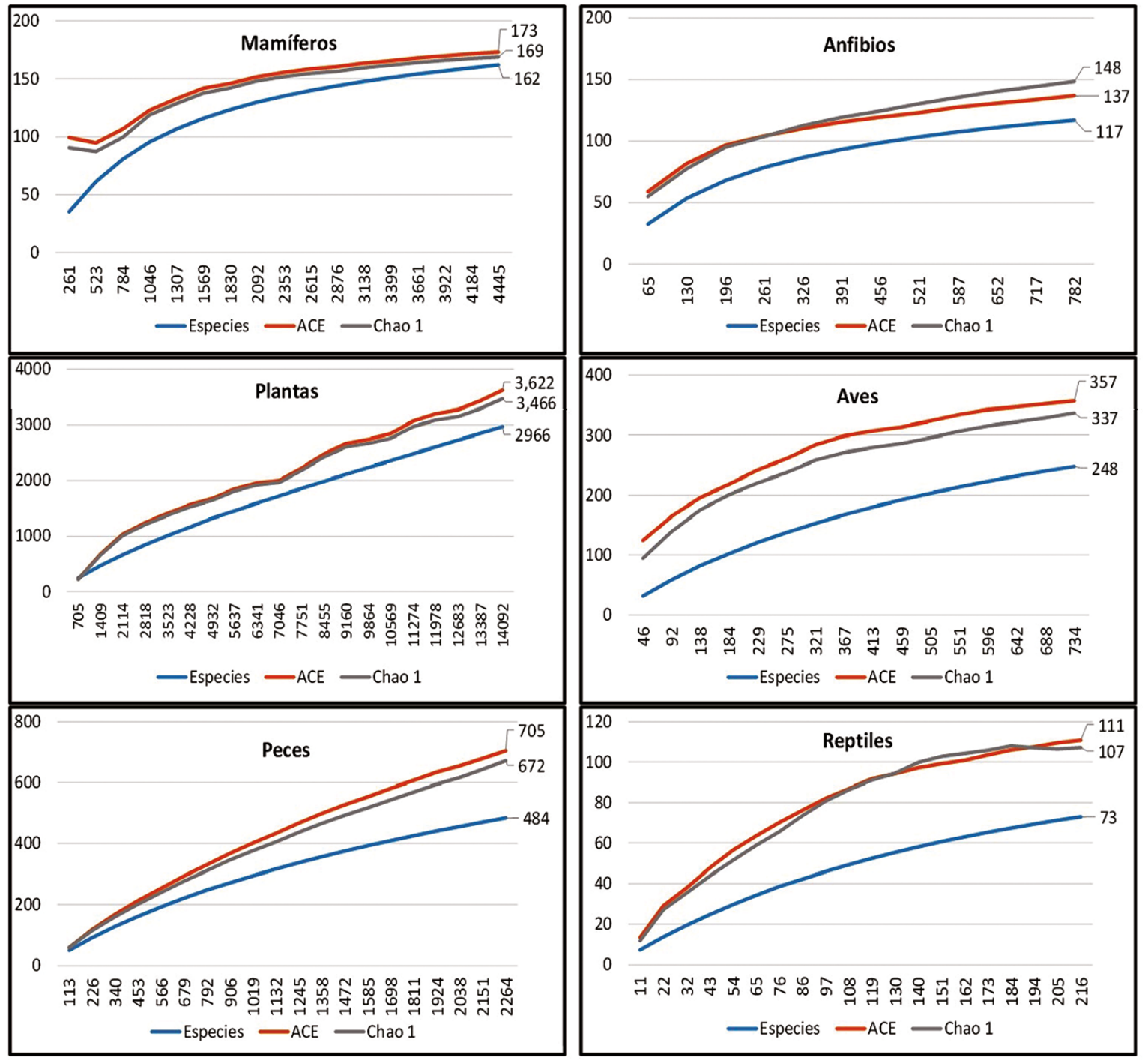

Figura 2. Curvas de acumulación de especies observadas y de los estimadores de riqueza ACE y Chao1 para los seis taxones estudiados, dentro de los $20 \mathrm{~km}$ de distancia a la carretera proyectada. El eje vertical muestra número de especies; el horizontal muestra el número de individuos muestreados.

Entre los mayores impactos de las carreteras identificados se encuentran la pérdida de hábitat y la cacería. En zonas con poco acceso y sin grandes poblaciones humanas estos dos fenómenos no suelen ser graves, pero, al facilitar el acceso, la apertura de carreteras atrae el asentamiento de poblaciones, creación de caminos y deforestación, pérdida de biodiversidad, desplazamiento de comunidades indígenas y reducción del almacenamiento de carbono (Vilela et al. 2020). Las nuevas carreteras y comunidades humanas facilitan el ingreso de cazadores a áreas antes inaccesibles y promueven el aumento del mercado de carne silvestre (Suárez et al. 
2009) como en el caso de Ecuador, donde se ha registrado una alta presión de caza hasta a $12 \mathrm{~km}$ de distancia del camino Maxus, incluso dentro de un área protegida (Espinosa et al. 2018).

En base a nuestras estimaciones de pérdida total de bosque en otras carreteras amazónicas peruanas (entre 1.64 y $4.65 \mathrm{~km}^{2}$ por $\mathrm{km}$ ), la deforestación asociada a la carretera Iquitos-Saramiriza oscilaría entre los 1128 y los $3200 \mathrm{~km}^{2}$ para los próximos 30 años, lo que equivale a entre el $8.2 \%$ y el $23.3 \%$ de la deforestación registrada en todo el departamento de Loreto en las últimas dos décadas $\left(13,760 \mathrm{~km}^{2}\right.$, Dourojeanni 2013, MINAM, 2018). Honorio et al. (2020) estimaron $7.05 \mathrm{~km}^{2}$ de deforestación por kilómetro para $350 \mathrm{~km}$ de esta misma vía (con $2468 \mathrm{~km}^{2}$ deforestados en total), lo que sugiere que la deforestación podría ser mayor de lo calculado aquí, en tramos específicos.

La pérdida de bosque, proyectada a 20 años, de hasta $17 \%$ dentro de la franja de $5 \mathrm{~km}$ a cada lado de la vía, muestra que la carretera Iquitos-Saramiriza podría convertirse en una barrera importante para la biodiversidad. En tramos en los que la deforestación se concentre más, la ausencia de árboles podría incluso limitar el paso de especies voladoras y de dispersores de semillas. La fragmentación podría afectar en menor o mayor grado a todas las especies registradas en su área de influencia, y ser especialmente trágica para la fauna cuya distribución se vería completamente (o casi completamente) cortada en dos por su trazo, en la cual destacan siete primates y 13 anfibios, animales que suelen evitar ambientes despejados de vegetación.
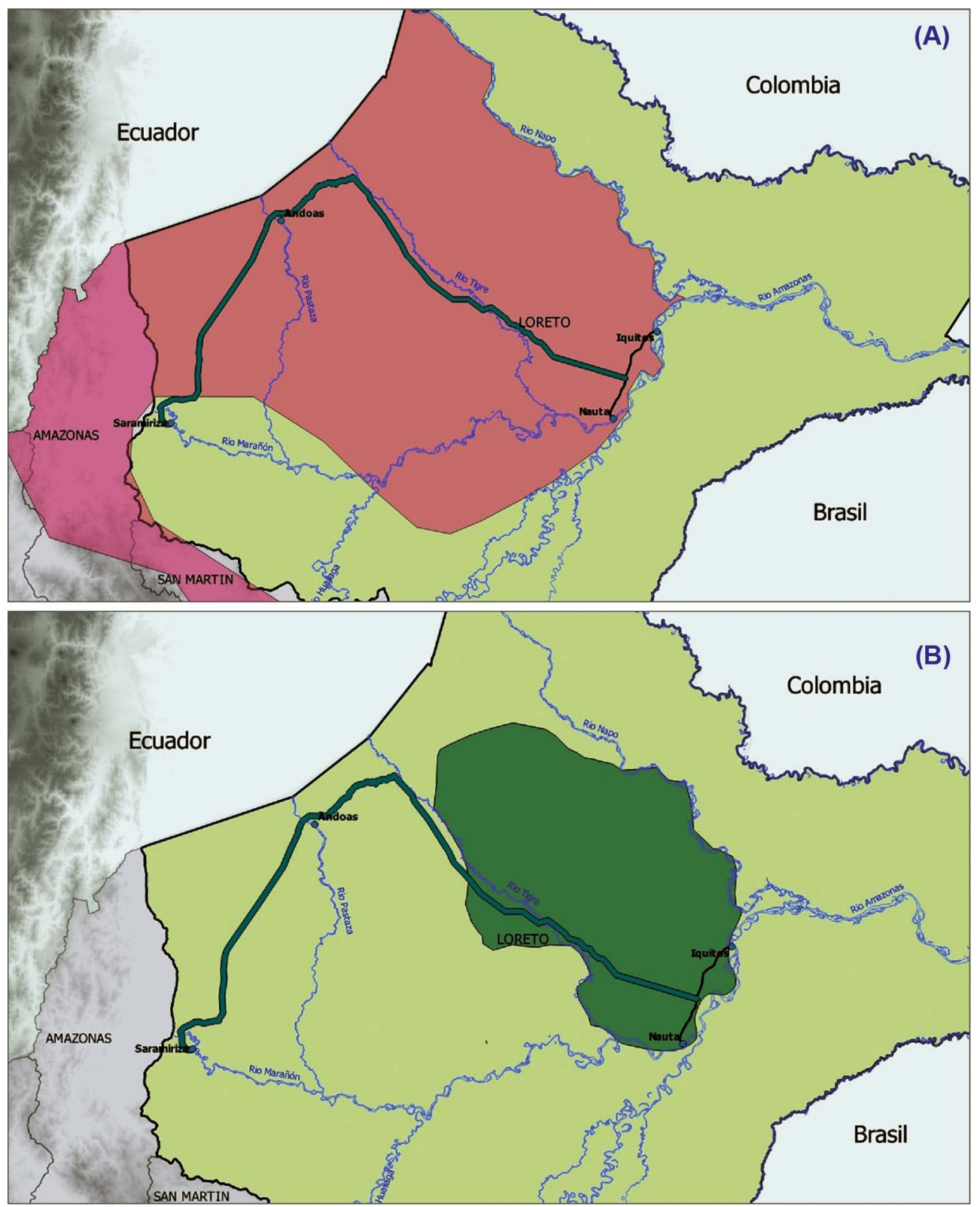

Figura 3. Trazo de la carretera lquitos-Saramiriza sobre el área de distribución de dos especies de primates, mostrando el posible efecto de fragmentación. A: Distribución en el Perú del mono araña grisáceo, Ateles belzebuth (en rosado), modificada de Link et al. (2019), B: Distribución del huapo ecuatorial, Pithecia aequatorialis (en verde oscuro), modificada de Marsh et al. (2019). En ambos casos, se muestra el departamento de Loreto en verde claro, el trazo de la carretera Iquitos-Saramiriza en verde y la carretera Iquitos-Nauta en negro. 
Como se ha mostrado en este trabajo, los impactos esperados de la carretera Iquitos-Saramiriza sobre la biodiversidad son múltiples y podrían ser graves si no se consideran medidas de mitigación. Considerando este panorama, es recomendable: a) evitar la construcción de tramos que puedan ser reemplazados por otras maneras con menor impacto y b) implantar medidas que disminuyan impactos en aquellos tramos que no puedan evitarse.

Tal como proponen Honorio et al. (2020), la construcción del tramo paralelo al río Tigre, de unos 349,63 $\mathrm{km}$, podría evitarse mejorando el transporte fluvial. Un sistema similar, con transporte fluvial subsidiado, fue propuesto para reemplazar una carretera entre Boca Manu y Boca Colorado, en el departamento de Madre de Dios (Gallice et al. 2017).

En donde la construcción sea inevitable, lo más recomendable es la creación de áreas de protección estricta a ambos lados de la carretera, para minimizar asentamientos, cacería y deforestación (Laurance et al. 2009, Fearnside 2006). Un ejemplo es la extensión del Parque Nacional costarricense Braulio Carrillo, en 1978, paralelamente a la carretera San José-Guápiles, lo que evitó la deforestación en esa zona (Ledec \& Posas 2003). La efectividad de las áreas protegidas para reducir la pérdida de bosques tropicales ha sido demostrada en diversas partes del mundo (Nagendra et al. 2008), incluyendo la Amazonía ecuatoriana (Van der Hoek 2017) y brasileña (Nolte et al. 2013). En Latinoamérica, sin embargo, el simple establecimiento de áreas protegidas no suele detener la deforestación (Leisher et al. 2013), siendo necesario establecer, además, medidas de protección, como un sistema efectivo de vigilancia, antes del trazado y construcción (Ledec y Posas 2003).

En las áreas de protección estricta, además, debe mitigarse la fragmentación facilitando el paso de fauna. Los puentes de dosel han mostrado ser particularmente efectivos para permitir el cruce de especies arborícolas sobre infraestructuras lineales como gasoductos y carreteras, mientras que en tramos sin estos puentes los cruces se reducen drásticamente, incluso desaparecen (Gregory et al. 2017) o puede haber importantes pérdidas poblacionales por atropellamiento (Donaldson y Cunneyworth 2014, Cunneyworth y Duke 2020). Para facilitar también el paso de especies de desplazamientos terrestres, se requieren pasos bajo tierra y pasos elevados (Smith et al. 2015, Myslajek et al. 2020), particularmente importantes para anfibios y reptiles, que suelen evitar espacios sin vegetación (Maynard et al. 2016).

Los puentes de dosel y los pasos terrestres de fauna contribuyen también a disminuir los atropellamientos. Además de estas medidas, la señalización, la definición de límites adecuados de velocidad y las campañas locales de educación pueden disminuir ese problema (van der Grift et al. 2013). Para aumentar su efectividad, la ubicación de puentes de dosel, pasos terrestres y señalización debe definirse con evaluaciones previas, que determinen en qué secciones del trazo de la carretera habrá mayor movimiento de animales (Ledec y Posas 2003, Gregory et al. 2017).

Siguiendo los puntos desarrollados arriba, las evaluaciones previas a la implementación del proyecto IquitosSaramiriza deberían incluir: a) evaluación de alternativas a distintos tramos, especialmente en el tramo paralelo al río Tigre, b) identificación de las mejores zonas para ubicar pasos de fauna, c) evaluación de biodiversidad, al menos hasta $20 \mathrm{~km}$ del trazo, con especial atención a la herpetofauna, por estar poco estudiada en el área y con altas probabilidades de presentar especies amenazadas, endémicas o nuevas, d) identificación de sitios para extracción de material de construcción, donde se minimice impactos ambientales, e) inventario forestal en todo el trazo y f) planificación de áreas de protección estricta, asegurando su vigilancia y control, especialmente entre los puntos 12 de Octubre y Saramiriza.

Además de la línea base con los resultados de las evaluaciones citadas arriba y de los planes usuales de mitigación de impactos durante la construcción y funcionamiento, el Estudio de Impacto Ambiental (EIA) debería contener: a) plan de ahuyentamiento y rescate de fauna, b) plan de monitoreo de abundancia y riqueza de biodiversidad, de deforestación, cacería y de presencia de especies invasoras, c) plan de evaluación del impacto de actividades de construcción sobre medios acuáticos y terrestres, d) plan de monitoreo y manejo de pasos de fauna a implementar, comparando la frecuencia relativa de cruces con lo observado durante los estudios previos, y mejorando o multiplicando los pasos de fauna si fuera necesario, e) plan de monitoreo de atropellamientos y medidas de mitigación en caso necesario. En países amazónicos como el Perú, los EIA de carreteras suelen restringirse a los impactos directos de la vía, obviando las posibilidades de invasión de tierras, cacería ilegal, caminos secundarios y deforestación asociadas a estos problemas (Laurance et al. 2009, Reid y de Souza 2005). Para tener una idea real de los impactos que estos proyectos pueden acarrear y para poder tomar decisiones objetivas sobre la implementación de este y de otros proyectos de infraestructura, es necesario que los organismos estatales exijan la consideración de esos fenómenos al momento de evaluar impactos y la inclusión obligatoria de medidas de mitigación adecuadas.

\section{Literatura citada}

Aguilar CA, Rojas-Padilla O, Rios-Alva EJ, Odicio-Iglesias MM, Aguilar-Manihuari R, Gagliardi-Urrutia G. 2021. Lista actualizada de los anfibios del departamento de Loreto. Revista Peruana de Biología 28 (especial):e21912. https://doi.org/10.15381/rpb.v28iespecial.21912

Aguilar CA, Rojas-Padilla 0, Rios-Alva EJ, Odicio-Iglesias MM, Aguilar-Manihuari R, Gagliardi-Urrutia G. 2021. Lista actualizada de los reptiles del departamento de Loreto. Revista Peruana de Biología 28 (especial):e21913. https://doi.org/10.15381/rpb.v28iespecial.21913.

Brum TR, Santos-Filho M, Canale GR, Ignácio ARA. 2018. Effects of roads on thevertebrates diversity of the Indigenous Territory Paresi and its surrounding. Brazilian Journal of Biology 78(1): 125-132. https://doi. org/10.1590/1519-6984.08116 
Cáceres NC, Hannibal W, Freitas DR, Silva EL, Roman C, Casella, J. 2010. Mammal occurrence and roadkill in two adjacent ecoregions (Atlantic Forest and Cerrado) in south-western Brazil. Zoologia 27:709-717. https:// doi.org/10.1590/S1984-46702010000500007

Ceballos G, Ehrlich PR. 2006. Global mammal distributions, biodiversity hotspots, and conservation. Proceedings of the National Academy of Sciences 103:19374-19379. https://doi.org/10.1073/pnas.0609334103

Colwell RK. 2013. EstimateS: Statistical estimation of species richness and shared species from samples. Version 9.1.0. < purl.oclc.org/estimates>

Cunneyworth PM, Duke J. 2020. Vehicle colissions among four species of monkeys between 2000 and 2018 on a suburban road in Diani, Kenya. International Journal of Primatology 41:45-60. https://doi.org/10.1007/ s10764-020-00135-w

Dar PA, Reshi ZA, Shah MA. 2015. Roads act as corridors for the spread of alien plant species in the mountainous regions: a case study of Kashmir Valley, India. Tropical Ecology 56(2):183-90.

Diaz S, Sánchez-Vendizú P, Graham-Angeles L, Pacheco V. 2021. Diversidad y conservación de los mamíferos mayores de Loreto, Perú. Revista Peruana de Biología 28 (especial):e21910. https://doi.org/10.15381/rpb. v28iespecial.21910.

Donaldson A, Cunneyworth P. 2015. Case study: canopy bridges for primate conservation. En: Van der Ree R, Smith DJ, Grilo C (Eds.), Handbook of road ecology. John Wiley and Sons Ltd, pp. 341-343. https://doi. org/10.1002/9781118568170.ch41

Dourojeanni M. 2013. Loreto sostenible al 2021. Derecho, Ambiente y Recursos Naturales - DAR, Lima, Perú. 356 pp.

Espinosa S, Celis G, Branch LC. 2018. When roads appear jaguars decline: Increased access to an Amazonian wilderness area reduces potential for jaguar conservation. PLoS ONE 13(1):e0189740. https://doi.org/10.1371/journal.pone.0189740

Famer, A.M. 1993. The effects of dust on vegetation - A review. Environmental Pollution 79:63-75. https://doi. org/10.1016/0269-7491(93)90179-R

Fearnside PM. 2006. Containing destruction from Brazil's Amazon highways: now is the time to give weight to the environment in decision-making. Environment Conservation 33:181-183. https://doi.org/10.1017/ S0376892906003109

Fearnside PM. 2015. Highway construction as a force in destruction of the Amazon forest. En: van der Ree R, Smith DJ, Grilo C. (Eds.), Handbook of Road Ecology. John Wiley \& Sons Publishers, Oxford, UK, pp. 414424. https://doi.org/10.1002/9781118568170.ch51

Fearnside PM. 1987. Causes of deforestation in the Brazilian Amazon. En: Dickinson RF. (Ed.), The Geophysiology of Amazonia: Vegetation and Climate Interactions. John Wiley \& Sons, New York, pp. 37-61.

Fensome, A.G. \& Mathews F. 2016 Roads and bats: a metaanalysis and review of the evidence on vehicle collisions and barrier effects. Mammal Review 46:311323 https://doi.org/10.1111/mam.12072

Filius J, van der Hoek Y, Jarrín-V P, van der Hooft P. 2020. Wildlife roadkill patterns in a fragmented landscape of the western Amazon. Ecology and Evolution 10:66236635. https://doi.org/10.1002/ece3.6394
Finer M, Jenkins CN, Pimm SL, Keane B, Ross C. 2008. Oil and gas projects in the western Amazon: threats to wilderness, biodiversity, and indigenous peoples. PLoS One. 3(8):e2932. https://doi.org/10.1371/journal. pone. 0002932

Finer M, Mamani N. 2020. Deforestación en la Amazonía 2019. MAAP (PROYECTO MONITOREO DE LA AMAZONÍA ANDINA): 122.

Frohn RC, Dale VH, Jimenez BD. 1990. Colonization, road development and deforestation in the Brazilian Amazon Basin of Rondonia. Environmental Science Divison Publication \# 3394. Oak Ridge Nat.Lab. Tennessee, EEUU. https://doi.org/10.2172/6946370

Gad, AM., Feinsod, F.M., Allam, I.H., Eisa, M., Hassan, A.N., Soliman, B.A., el Said, S. \& Saah, A.J. 1986. A possible route for the introduction of Rift Valley fever virus into Egypt during 1977. J. Trop. Med. Hyg. 89(5):233-236. https://doi.org/10.1016/j.actatropica.2018.01.015

Gallice GR, Larrea-Gallegos G, Vásquez-Rowe I. 2017. The threat of road expansion in the Peruvian Amazon. Oryx 53(2): 284-292. https://doi.org/10.1017/ S0030605317000412

Google earth V 7.3.3.7786 (64-bit) Jul 2020. http://www.earth. google.com

Goosem M. 2002. Effects of tropical rainforest roads on small mammals: fragmentation, edge effects and traffic disturbance. Wildlife Research 29: 277-289. https://doi. org/10.1071/WR01058

Graham-Angeles L, Sánchez-Vendizú P, Diaz S, Pacheco V. 2021. Diversidad y conservación de los murciélagos de Loreto, Perú. Revista Peruana De Biología 28 (especial):e21917. https://doi.org/10.15381/rpb. v28iespecial.21917.

Gregory T, Carrasco-Rueda F, Alonso A, Kolowski J, Deichmann JL. 2017. Natural canopy bridges effectively mitigate tropical forest fragmentation for arboreal mammals. Nature Scientific Reports 7:3892. https://doi. org/10.1038/s41598-017-04112-x

Heigl, F., Horvath, K., Laaha, G. et al. 2017. Amphibian and reptile road-kills on tertiary roads in relation to landscape structure: using a citizen science approach with openaccess land cover data. BMC Ecol 17, 24. https://doi. org/10.1186/s12898-017-0134-z

Honorio EN, Mercado A, Del Castillo D, Dávila N, Martín M, Ríos S, Baker TR, Montoya M. 2020. Impacto de la construcción de la carretera Iquitos-Saramiriza sobres los bosques y turberas del Río Tigre, Loreto, Perú. Folia Amazónica. 29(1): 65-87. https://doi.org/10.24841/ fa.v28i2.493

Howard C, Flather CH, Stephens PA. 2020. A global assessment of the drivers of threatened terrestrial species richness. Nature Communications 11: 993. https://doi. org/10.1038/s41467-020-14771-6

Imbernon J. 1999. A comparison of the driving forces behind deforestation in the Peruvian and the Brazilian Amazon. Ambio 28: 509-513.

Jochimsen DM, Peterson CR, Andrews KM, Whitfield GJ. 2004. A Literature Review of the Effects of Roads on Amphibians and Reptiles and the Measures Used to Minimize Those Effects. Idaho Fish and Game Department. USDA Forest Service. 
Kaimowitz D. 1997. Factors determining low deforestation in the Bolivian Amazon. Ambio 26: 537-540. https:// www.cifor.org/knowledge/publication/5

Khamcha,D., Powell,L.A., Gale, G.A. 2018. Effects of roadside edge on nest predators and nest survival of Asian tropical forest birds. Global Ecology and Conservation 16 (2018) e00450. https://doi.org/10.1016/j.gecco.2018.e00450

Killeen TJ, Douglas M, Consiglio T, Jørgensen PM, Meika J. 2007. Dry spots and wet spots in the Andean hotspot. Journal of Biogeography 34: 1357-1373. https://doi. org/10.1111/j.1365-2699.2006.01682.x

Laurance WF, Gossem M, Laurance SGW. 2009. Impacts of roads and linear clearings on tropical forests. Trends in Ecology and Evolution 24(12):659-669. https://doi. org/10.1016/j.tree.2009.06.009

Laurance WF, Clements GR, Sloan S, O'Connell CS, Mueller ND, Goosem M, Venter O, Edwards DP, Phalan B, Balmford A, van Der Ree R, Arrea IB. 2014. A global strategy for road building. Nature 513:229-234. https://doi. org/10.1038/nature13717

Ledec G, Posas PJ. 2003. Biodiversity Conservation in Road Projects: Lessons from World Bank Experience in Latin America. Transportation Research Record Journal of the Transportation Research Board 1819(1): 198202. https://doi.org/10.3141/1819a-29

Leisher C, Touval J, Hess SM, Boucher TM, Reymondin L. 2013. Land and forest degradation inside protected areas in Latin America. Diversity 5: 779-795. https://doi. org/10.3390/d5040779

Link A, Muniz C, Rylands AB, Mourthé Í, Cornejo FM, Urbani B, Mittermeier RA, Stevenson PR, Palacios E, Boubli J, Shanee S, de la Torre S, Moscoso P. 2019. Ateles belzebuth. The IUCN Red List of Threatened Species 2019: e.T2276A17928557. https://doi.org/10.2305/IUCN. UK.2019-2.RLTS.T2276A17928557.en

Marjakangas EL, Abrego N, Groten V, de Lima RAF, et al. 2019. Fragmented tropical forests lose mutualistic plantanimal interactions. Diversity and Distributions 26(2): 154-168. https://doi.org/10.1111/ddi.13010

Marsh LK, Heymann EW. 2018. Pithecia aequatorialis. The IUCN Red List of Threatened Species 2018: e.T17402A17971831. https://doi.org/10.2305/ IUCN.UK.2018-2.RLTS.T17402A17971831.en

Marsik M, Stevens FR, Southworth J. 2011. Amazon deforestation: Rates and patterns of land cover change and fragmentation in Pando, northern Bolivia, 1986 to 2005. Progress in Physical Geography 35(3): 353-374. https://doi.org/10.1177/0309133311399492

Maynard RJ, Aall NC, Saenz D, Hamilton PS, Kwiatkowski MA. 2016. Road-edge effects on herpetofauna in a lowland Amazonian rainforest. Tropical Conservation Science 9(1): 264-290. https://doi. org/10.1177/194008291600900114

Mena CF, Bilsborrow RE, McClain ME. 2006. Socioeconomic drivers of deforestation in the northern Ecuadorian Amazon. Environmental Management 37: 802-815. https://doi.org/10.1007/s00267-003-0230-z

Meza-Vargas V, Faustino-Fuster DR, Chuctaya J, Hidalgo M, Ortega Torres H. 2021. Lista de especies de peces de agua dulce de Loreto, Perú. Revista Peruana de Biología 28 (especial):e21911. https://doi.org/10.15381/ rpb.v28iespecial.21911.

MINAM 2018. Cobertura y deforestación en los bosques húmedos amazónicos 2018. (C) Ministerio del Ambien- te (MINAM). Programa Nacional de Conservación de Bosques para la Mitigación del Cambio Climático (PNCBMCC).

Monroy-Vilchis O, González-Maya JF, Balbuena-Serrano Á, Elvir F, Zarco-González MM, Rodríguez-Soto C. 2020. Coyote (Canis latrans) in South America: potential routes of colonization. Integrative Zoology. 15(6):471-481. https://doi.org/10.1111/1749-4877.12446

Mysłajek RW, Olkowska E, Wronka-Tomulewicz M, et al. Mammal use of wildlife crossing structures along a new motorway in an area recently recolonized by wolves. European Journal of Wildlife Research 66: 79. https:// doi.org/10.1007/s10344-020-01412-y

Nagendra H. 2008. Do parks work? Impact of protected areas on land cover clearing. Ambio 37: 330-337. https:// doi.org/10.1579/06-R-184.1

Nolte C, Agrawal A, Silvius KM, Soares-Filho BS. 2013. Governance regime and location influence avoided deforestation success of protected areas in the Brazilian Amazon. Proceedings of the National Academy of Sciences 110: 4956-4961. https://doi.org/10.1073/ pnas. 1214786110

Noss RF, Quigley HB, Hornocker MG, Merrill T, Paquet PC. 1996. Conservation biology and carnivore conservation in the Rocky Mountains. Conservation Biology 10:949-963. https://doi.org/10.1046/j.15231739.1996.10040949.x

Oliveira PJC, Asner GP, Knapp DE, Almeyda A, Galván-Gildemeister R, Keene S, Raybin RF, Smith RC. 2007. LandUse Allocation Protects the Peruvian Amazon. Science 317(5842):1233-1236. https://doi.org/10.1126/ science.1146324

Perz SG, Overdevest C, Arima EY, Caldas MM, Walker RT. 2007. Unofficial Road Building in the Brazilian Amazon: Dilemmas and Models of Road Governance. Environmental Conservation 34(2): 112-121. https://doi. org/10.1017/S0376892907003827

Perz S, Brilhante S, Brown F, Caldas M, Ikeda S, Mendoza S, Overdevest C, Reis V, Reyes JF, Rojas D, Schmink M, Souza C, Walker R. 2008. Road building, land use and climate change: prospects for environmental governance in the Amazon. Philosophical Transactions of the Royal Society B. 363:1889-1895. https://doi.org/10.1098/ rstb.2007.0017

Perz SG. 2014. Sustainable development: the promise and perils of roads. Nature 513: 178-179. https://doi. org/10.1038/nature13744

Pfaff A, Robalino J, Walker R, Aldrich S, Caldas M, Reis E, et al. 2007. Road investments, spatial spillovers and deforestation in the Brazilian Amazon. Journal of Regional Science 47(1):109-123. https://doi.org/10.1111/ j.1467-9787.2007.00502.x

Pielou EC. 1969. An introduction to mathematical ecology. Wiley-Interscience, New York.

Rieux, A., Soubeyrand, S., Bonnot, F., Klein, E.K. et al. 2014 Longdistance wind-dispersal of spores in a fungal plant pathogen: estimation of anisotropic dispersal kernels from an extensive field experiment. PLoS ONE 9, e103225 (2014). doi: 10.1371/journal.pone.0103225

Salinas L, Arana A, Arana C. 2021. Las aves del departamento de Loreto, Perú. Revista Peruana de Biología 28 (especial):e21915. https://doi.org/10.15381/rpb. v28iespecial.21915.

Sánchez-Vendizú P, Graham-Angeles L, Diaz S, Pacheco V. 2021. Diversidad y estado de conservación de pequeños ma- 
míferos de Loreto, Perú. Revista Peruana de Biología 28 (especial):e21907. https://doi.org/10.15381/rpb. v28iespecial.21907..

SERFOR. 2018. Libro Rojo de la Fauna Silvestre Amenazada del Perú. Primera edición. Lima. 532 pp.

Smith DJ, van der Ree R, Rosell C. 2015. Wildlife crossing structures: an effective strategy to restore or maintain wildlife connectivity across roads. En: van der Ree R, Smith DJ, Grilo C. (Eds), Handbook of road ecology. John Wiley and Sons, Chichester. https://doi. org/10.1002/9781118568170

Soares BS, Nepstad DC, Curran LM, Cerqueira GC, Garcia RA, et al. 2006. Modeling conservation in the Amazon basin. Nature 440: 520-523. https://doi.org/10.1038/nature04389

Suárez E, Morales M, Cueva R, Utreras-Bucheli V, Zapata-Ríos G, Toral E, Torres J, Prado W, Vargas Olalla J. 2009. Oil industry, wild meat trade and roads: indirect effects of oil extraction activities in a protected area in northeastern Ecuador. Animal Conservation. 12:364-373. https://doi.org/10.1111/j.1469-1795.2009.00262.x

Trombulak SC, Frissell CA. 2000. Review of ecological effects of roads on terrestrial and aquatic communities. Conservation Biology 14: 18-30. https://doi.org/10.1046/ j.1523-1739.2000.99084.x

Valladares-Padua, C., Cullen Junior, L. \& Ppadua, S. 1995. A pole bridge to avoid primate road kills. Neotrop. Primates 3(1):13-15. DOI: 10.1590/S167606032013000100013

Van der Oek 2017. The potential of protected areas to halt deforestation in Ecuador Environmental Conservation 44(2):1-7. DOI:10.1017/S037689291700011X
Van der Grift E.A., van der Ree, R., Fahrig, L., Findlay, S., Houlahan, J., Jaeger, J.A.G., Olson. L.. 2013. Evaluating the effectiveness of road mitigation measures. Biodiversity and Conservation 22: 425-448. https://doi. org/10.1007/s10531-012-0421-0

Vilela T, Harb AM, Bruner A, Da Silva Arruda VL, Ribeiro V, Alencar AAC, Escobedo-Grandez AJ, Rojas A, Laina A, Botero R. 2020. A better Amazon road network for people and the environment. Proceedings of the National Academy of Sciences. 117(13): 7095-7102. https:// doi.org/10.1073/pnas.1910853117

Volpe N.L., Robinson W.D., Frey S.J.K., Hadley A.S., Betts M.G. 2016. Tropical forest fragmentation Limits Movements, but Not Occurrence of a Generalist Pollinator Species. PLoS ONE 11(12): e0167513. https://doi. org/10.1371/journal.pone.0167513

Whitford, P. C. 1985. Bird behavior in response to the warmth of blacktop roads. Transactions of the Wisconsin Academy of Sciences Arts and Letters 73:135-143. https://www.jstor.org/stable/27976457

Yesquen SF, Ugaz CAF, Chávez VCL. 2020, Mortandad de vertebrados por atropellos en carreteras en Tambogrande, Piura, Perú. Revista Peruana de Biología 27(2):131.138. https://doi.org/10.15381/rpb. v27i2.16827

\section{Agradecimientos / Acknowledgments:}

Queremos agradecer a César Aguilar, Víctor Pacheco, Letty Salinas, Vanessa Meza y Ricardo Zárate, que proporcionaron valiosos datos georreferenciados. Tania Galván ayudó con los análisis geográficos, Mical Veillon en la preparación de bases de datos y Andrew Noss revisó el manuscrito y proporcionó valiosos comentarios. Esta publicación contó con el apoyo económico de WCS-Perú.

Conflicto de intereses / Competing interests:

Los autores no incurren en conflictos de intereses.

\section{Rol de los autores / Authors Roles:}

LM: Conceptualización, Investigación, Escritura- Preparación del borrador original, Redacción: revisión y edición. EDC: Investigación, Escritura - Preparación del borrador original, Redacción: revisión y edición,.

\section{Fuentes de financiamiento / Funding:}

Este trabajo contó con el financiamiento de Wildlife Conservation Society-Perú.

Aspectos éticos / legales; Ethics / legals:

Los autores declaran no haber incurrido en ningún aspecto antiético ni omitido norma legal nacional o internacional en la investigación y elaboración del articulo. 
Anexo 1. Especies susceptibles a la presión de caza debido a mejor acceso a las zonas silvestres.

\begin{tabular}{|c|c|c|c|}
\hline Mamíferos & Endemismo & Amenaza Peru & Amenaza Global \\
\hline \multicolumn{4}{|l|}{ Mamíferos } \\
\hline Alouatta seniculus & & VU & \\
\hline Ateles belzebuth & & EN & EN \\
\hline Ateles chamek & & EN & EN \\
\hline Cacajao calvus & & VU & VU \\
\hline \multicolumn{4}{|l|}{ Callicebus discolor } \\
\hline Callicebus lucifer & & VU & \\
\hline Callimico goeldii & & VU & VU \\
\hline \multicolumn{4}{|l|}{ Cebus (Cebus) albifrons } \\
\hline \multicolumn{4}{|l|}{ Cebus (Sapajus) apella } \\
\hline Inia geoffrensis & & & EN \\
\hline Lagothrix lagothricha & & EN & VU \\
\hline Lagothrix poeppigii & & EN & VU \\
\hline \multicolumn{4}{|l|}{ Mazama americana } \\
\hline \multicolumn{4}{|l|}{ Mazama nemorivaga } \\
\hline Pithecia aequatorialis & Perú & & \\
\hline Priodontes maximus & & VU & VU \\
\hline \multicolumn{4}{|l|}{ Saguinus (Leontocebus) lagonotus } \\
\hline Saguinus (Leontocebus) nigrifrons & Perú & & \\
\hline Saguinus (Leontocebus) tripartitus & & VU & \\
\hline \multicolumn{4}{|l|}{ Saguinus (Tamarinus) mystax } \\
\hline \multicolumn{4}{|l|}{ Saimiri boliviensis } \\
\hline \multicolumn{4}{|l|}{ Saimiri sciureus } \\
\hline Tapirus terrestris & & & VU \\
\hline Tayassu pecari & & & VU \\
\hline Trichechus inunguis & & VU & VU \\
\hline \multicolumn{4}{|l|}{ Aves } \\
\hline \multicolumn{4}{|l|}{ Crypturellus cinereus } \\
\hline \multicolumn{4}{|l|}{ Crypturellus undulatus } \\
\hline \multicolumn{4}{|l|}{ Ortalis gutatta } \\
\hline \multicolumn{4}{|l|}{ Penelope jacquacu } \\
\hline \multicolumn{4}{|l|}{ Tinamus guttatus } \\
\hline Tinamus major & & & \\
\hline
\end{tabular}

